\title{
Article \\ Fibril Growth Behavior of Amyloid $\beta$ on Polymer-Based Planar Membranes: Implications for the Entanglement and Hydration of Polymers
}

\author{
Toshinori Shimanouchi *, Miki Iwamura, Shintaro Deguchi and Yukitaka Kimura \\ Graduate School of Environment and Life Science, Okayama University, 3-1-1 Tsushimanaka, Kita-Ku, \\ Okayama 700-8530, Japan; ev422406@s.okayama-u.ac.jp (M.I.); pjua4wdz@s.okayama-u.ac.jp (S.D.); \\ yktkkimu@cc.okayama-u.ac.jp (Y.K.) \\ * Correspondence: tshima@cc.okayama-u.ac.jp; Tel.: +81-86-251-8908
}

check for updates

Citation: Shimanouchi, T.; Iwamura, M.; Deguchi, S.; Kimura, Y. Fibril Growth Behavior of Amyloid $\beta$ on Polymer-Based Planar Membranes: Implications for the Entanglement and Hydration of Polymers. Appl. Sci. 2021, 11, 4408. https://doi.org/ 10.3390/app11104408

Academic Editor:

Alejandro Rodriguez Pascual

Received: 5 April 2021

Accepted: 8 May 2021

Published: 13 May 2021

Publisher's Note: MDPI stays neutral with regard to jurisdictional claims in published maps and institutional affiliations.

Copyright: (c) 2021 by the authors. Licensee MDPI, Basel, Switzerland. This article is an open access article distributed under the terms and conditions of the Creative Commons Attribution (CC BY) license (https:/ / creativecommons.org/licenses/by/ $4.0 /)$.
Featured Application: Biocompatible materials in artificial organs, biosensors, or modifiers for a vehicle in a drug delivery system.

Abstract: The design of biosensors and artificial organs using biocompatible materials with a low affinity for amyloid $\beta$ peptide $(\mathrm{A} \beta$ ) would contribute to the inhibition of fibril growth causing Alzheimer's disease. We systematically studied the amyloidogenicity of $A \beta$ on various planar membranes. The planar membranes were prepared using biocompatible polymers, viz., poly(methyl methacrylate) (PMMA), polysulfone (PSf), poly(L-lactic acid) (PLLA), and polyvinylpyrrolidone (PVP). Phospholipids from biomembranes, viz., 1,2-dioleoyl-phosphatidylcholine (DOPC), 1,2dipalmitoyl-phosphatidylcholine (DPPC), and polyethylene glycol-graft-phosphatidyl ethanolamine (PEG-PE) were used as controls. Phospholipid- and polymer-based membranes were prepared to determine the kinetics of $A \beta$ fibril formation. Rates of $A \beta$ nucleation on the PSf- and DPPC-based membranes were significantly higher than those on the other membranes. A $\beta$ accumulation, calculated by the change in frequency of a quartz crystal microbalance (QCM), followed the order: PSf $>$ PLLA > DOPC > PMMA, PVP, DPPC, and PEG-PE. Nucleation rates exhibited a positive correlation with the corresponding accumulation (except for the DPPC-based membrane) and a negative correlation with the molecular weight of the polymers. Strong hydration along the polymer backbone and polymer-A $\beta$ entanglement might contribute to the accumulation of $\mathrm{A} \beta$ and subsequent fibrillation.

Keywords: biocompatible polymer; amyloid fibril; amyloid $\beta$; nucleation; quartz crystal microbalance; hydration; entanglement; hydration

\section{Introduction}

Recent developments in medical devices, including artificial organs and biosensors, have focused on the inhibition of protein accumulation or adsorption. This is because the deposition of protein on the surface often leads to the deterioration of artificial organs due to surface contamination or the development of human amyloidosis. For example, the dialyzer, an artificial organ, is used to filter $\beta_{2}$-microglobulin $\left(\beta_{2}-\mathrm{mg}\right)$ in dialysis-related amyloidosis (DA) [1,2] and amyloid $\beta$ peptides $(A \beta)$ in Alzheimer's disease (AD) [3] from the bloodstream. However, $\beta_{2}-\mathrm{mg}$ and $A \beta$ accumulate on the surface of dialyzing membranes to induce amyloidosis [1]. Likewise, the biosensor also requires the suppression of adsorption of pathological proteins such as $\beta_{2}-\mathrm{mg}, \mathrm{A} \beta$, and $\alpha$-synuclein ( $\alpha$-syn) in Parkinson's disease, as well as serum albumin. Therefore, robust operation of medical devices requires effective inhibition of protein adsorption.

Many hydrophilic polymers developed for this purpose are biocompatible. Poly (vinylpyrrolidone) (PVP) [4,5], poly(L-lactic acid) (PLLA) [6,7], poly(methyl methacrylate) 
(PMMA) [8], poly(sulfonate) (PSf) [9], and others [10] are well-known examples. Several homopolymers have been used to produce blends [11,12] and block copolymers [13]. Nanoscopic architectures using these polymers, viz., planar membranes [7], membranes with asymmetric pore structures [14], (nano)particles [15], polymer brushes [16], and vesicles (polymersomes) [13] have been reported. From the perspective of biocompatibility, the surfaces of these polymeric materials require hydrophilicity or the reduction in protein adsorption [16].

With the development of polymer engineering, the pathological mechanisms of amyloidosis, such as DA [2], AD [17,18], and Parkinson's disease [19], have been revealed in the past two decades. Furthermore, amyloid fibril formation, as the key process in amyloidosis, has been clarified at the molecular level $[2,20]$. The mechanisms of fibril formation for $\beta_{2}$-mg, $A \beta$, and $\alpha$-syn are similar, i.e., fibril formation proceeds by nucleation and subsequent elongation [2]. Of these, only $A \beta$ is addressed in this study.

$\mathrm{A} \beta$ is a soluble protein with a $4.5 \mathrm{kDa}$ peptide related to $\mathrm{AD}$ [20]. $\mathrm{A} \beta$ aggregation on neuronal cell membranes is a hallmark of $\mathrm{AD}$ [18]. Recent studies have demonstrated a variety of molecular self-assemblies of $\mathrm{A} \beta$, including oligomers [21], micellar aggregates [22], protofibrils [23], and fibrillar aggregates, including spherulitic fibrillar aggregates (spherulite) [24-27]. At most, ten monomeric $A \beta$ form an oligomer on the biomembrane, and indicate strong cytotoxicity [21]. Oligomers bind with monomeric $A \beta$ to grow up to protofibrils, eventually forming mature fibrils (fibrillar aggregates) [21]. In addition, spherulites have a structure similar to senile plaques on neuronal cell membranes [24]. These morphologies can be induced on the phospholipid membranes, polymer membranes, and others [21,24-29]. Hence, interaction of external factors with $A \beta$ fibril formation affords a variety of morphologies of $A \beta$ aggregates. Thus, $A \beta$ fibril formation is the result of the contribution of external environments, including the interfaces of lipid and polymer membranes, i.e., the partitioning of $A \beta$ into the interfaces.

Electrostatic interaction [30,31], hydrophobic interaction [27,32], or topological interaction [33,34] are possible mechanisms of $A \beta$ partitioning into the interfaces. For polymer-based interfaces, the topological interaction between $A \beta$ peptides and polymers should be considered. This is because, in an earlier study, the partitioning behavior of peptides in the biphasic water/polymer solution system was determined by the entanglement of peptides with the polymer [34]. For example, poly(ethylene oxide) (PEO), having a molecular weight $(M w)$ of 5,000,000, entangled with proteins, contributing to their partitioning [34]. The entanglement features of polymers determine peptide partitioning as well as polymer-polymer interaction [35]. The entanglement of polymers or polymerpeptide originates from the fact that polymers with long-chain structures cannot cross over each other. In the field of polymer chemistry and physics, the entanglement of polymers has been accordingly characterized by the entanglement molecular weight $\left(M_{\mathrm{e}}\right)$, which corresponds to the $M w$ between entanglements. The $M_{\mathrm{e}}$ value can be estimated from the viscoelastic modulus [11,36] and ${ }^{1} \mathrm{H}-\mathrm{NMR}$ [37].

The physicochemical properties of polymers at the interface are generally different from those of the bulk polymer phase [38]. This is (i) because polymers in the random coiled state collapse, and thus, charge a higher elastic energy, and (ii) because of the constraint of self-diffusion of polymers due to entanglement $[39,40]$. This property is prominent in polymer membranes prepared by the layer-by-layer method [41]. If this effect would result in an interfacial environment that is advantageous for the binding of amphiphilic molecules, the induction of various morphologies of $A \beta$ fibrils [29] might be explained. However, the influence of the entanglement between polymers or between polymers and peptides on peptide binding is still unknown. It is anticipated that the influence of the entanglement of polymers at the interface would provide helpful insights into the development of biocompatible polymers for medical devices, such as artificial organs or biosensors, with low protein adsorption. 
In this study, we selected various polymers, viz., PVP, PLLA, PMMA, and PSf, as shown in Figure 1. These linear polymers were selected to discuss the effect of their $M_{\mathrm{e}}[5,38,39,42]$ (Table 1) and hydration structure [16,23,43-45]. PLLA with different $M w$ $(5000$ to 50,000$)$ was used to examine the effect of the entanglement of polymers while avoiding the influence of its chemical structure. PVP and PSf were selected as hydrophilic and hydrophobic standard polymers, respectively. Syndiotactic-PMMA (syn-PMMA) and isotactic-PMMA (iso-PMMA) were used to investigate the influence of the tacticity of the polymers. Phospholipids, viz., 1,2-dioleoyl-phosphatidylcholine (DOPC), 1,2-dipalmitoylphosphatidylcholine (DPPC), and phosphoethanolamine- $N$-[Methoxy(Polyethylene Glycol) 2000] (PEG-PE) were also used for comparison with polymer membranes, because the planar phospholipid membranes have been regarded as model biomembranes. In the first series of experiments, the fibril formation behavior of $A \beta$ on various planar polymer membranes was investigated in terms of the morphology of fibrils and kinetics according to previous reports [28] using total internal fluorescence microscopy (TIRFM) [29]. Kinetic analysis of fibril formation was performed according to a previous report [28] to obtain the kinetic parameters, such as the nucleation rate. The nucleation rate was compared with $A \beta$ accumulation on the planar membrane. Here, $A \beta$ accumulation was estimated using a quartz crystal microbalance (QCM) following immobilization of planar membranes [23]. Finally, we discussed the interaction of $A \beta$ with planar polymer membranes in terms of entanglement and hydration structure.

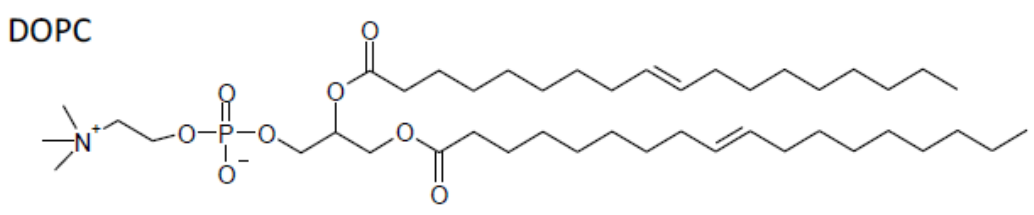

DPPC<smiles>CCCCCCCCCCCCCCCCCCCCCCCCC(=O)OCC(COP(=O)([O-])OCC[N+](C)(C)C)OC(=O)CCCCCC</smiles>

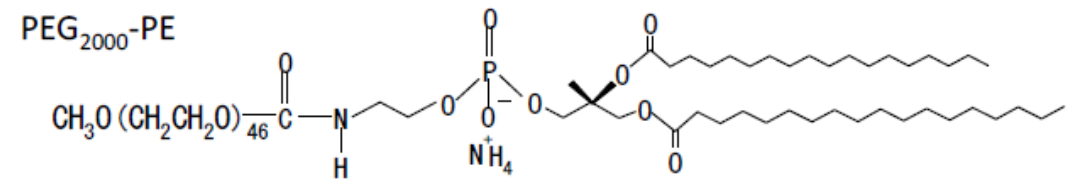
iso-PMMA syn-PMMA PVP<smiles>CCC(C)(C)CC(C)(C)C(C)(C)C</smiles><smiles>CCC(C)(C)CC(C)(C)C(C)(C)C(C)=O</smiles><smiles>CC(C)CN1CCCC1=O</smiles>

PSf

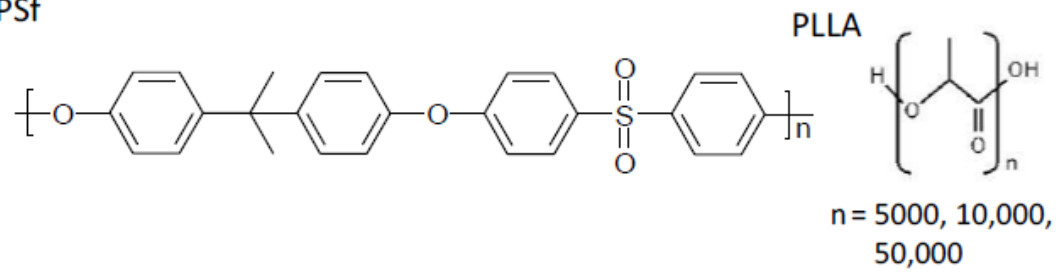

Figure 1. Chemical structure of phospholipids and polymers used herein. 
Table 1. Summary of polymers.

\begin{tabular}{cccc}
\hline Polymer & Molecular Weight, $\boldsymbol{M w}$ & $\begin{array}{c}\text { Entanglement } \\
\text { Molecular Weight, } \boldsymbol{M}_{\mathbf{e}}\end{array}$ & $\begin{array}{c}\text { Possible } \\
\text { Entanglement, } \boldsymbol{M}_{\boldsymbol{w}} / \boldsymbol{M}_{\mathbf{e}}\end{array}$ \\
\hline PVP & $1,200,000$ & $12,000^{*}$ & 100 \\
iso-PMMA & 500,000 & $14,600^{\#}$ & 34.2 \\
syn-PMMA & 400,000 & $9200^{\#}$ & 43.5 \\
PSf & 75,000 & $2250^{\%}$ & 33.3 \\
& 5000 & & 0.56 \\
PLLA & 10,000 & $9000^{\$}$ & 1.1 \\
& 20,000 & & 2.2 \\
\hline
\end{tabular}

${ }^{*}[38],{ }^{\%}[46],{ }^{\#}[47],{ }^{\$}[48]$.

\section{Materials and Methods}

\subsection{Materials}

DOPC and DPPC were obtained from Avanti Polar Lipids (Alabaster, AL, USA). PEGPE was obtained from Funakoshi Co., Ltd. (Tokyo, Japan). PLLA ( $M w=5000,10,000$, 20,000, and 50,000) was purchased from Wako Pure Chemical (Hiroshima, Japan). PMMA $(M w=400,000$ and 500,000), PSf $(M w=75,000)$, and PVP K90 $(M w=1,200,000)$ (supplied by Nacalai Tesque) were gifted by TORAY Co. Ltd. (Shiga, Japan).

$\mathrm{A} \beta$ with forty amino acid residues was purchased from the Peptide Institute (Osaka, Japan); its sequence was as follows: $\mathrm{H}_{2} \mathrm{~N}$-Asp-Ala-Glu-Phe-Arg-His-Asp-Ser-Gly-Tyr-GluVal-His-His-Gln-Lys-Leu-Val-Phe-Phe-Ala-Glu-Asp-Val-Gly-Ser-Asn-Lys-Gly-Ala-Ile-IleGly-Leu-Met-Val-Gly-Gly-Val-Val-COOH. Thioflavin T (ThT) for monitoring fibrillization was obtained from Dojindo (Kumamoto, Japan). All other reagents used were of analytical grade.

\subsection{Preparation of Planar Membranes Using Phospholipid and Polymers}

We prepared a planar phospholipid-based membrane as described previously [23,49]. Briefly, a thiol self-assembled membrane using 1-decane thiol was formed on an AT-cut quartz crystal with a gold electrode having $5.1 \mathrm{~mm}$ diameter. Next, this substrate was immersed in a DOPC/chloroform solution overnight to obtain a planar phospholipidbased membrane. The dried membrane was washed with distilled water at least three times to remove the excess lipid layers. Consequently, a DOPC monolayer was obtained on the thiol self-assemblies. The same was done for DPPC and PEG-PE.

For the polymer-based membrane, the above electrode was dipped into a $1 \%(w / w)$ polymer/chloroform solution for $24 \mathrm{~h}$. The dipped substrate was dried in vacuum and washed twice by double-distilled water. The spin-coating method was also used to form planar polymer-based membranes. An aliquot $(0.3 \mathrm{~mL})$ of $1 \%(w / w)$ polymer/chloroform solution was dispensed on the substrate attached to the spin coater. A rotation rate of 1000 was imposed on the substrate for $1 \mathrm{~min}$ so that the polymers could be fully stretched to form a surface with low roughness [50]. The resultant substrate was dried for $1 \mathrm{~h}$ at $60^{\circ} \mathrm{C}$. Here, drying the polymer solution at the edges of the substrate created ridges that affected the surface roughness and fibril formation behavior of $A \beta$. Hence, to maintain the quality of the data, the edge of the substrate was not used for other measurements.

\subsection{A $\beta$ Fibril Formation and Its Observation}

$\mathrm{A} \beta$ was dissolved in a $0.02 \%$ ammonia solution at a concentration of $200 \mu \mathrm{M}$ at $4{ }^{\circ} \mathrm{C}$. This solution was occasionally centrifuged at $10,000 \mathrm{rpm}$, at $4{ }^{\circ} \mathrm{C}$ for $20 \mathrm{~min}$, to separate the aggregates, because such aggregates can act as nuclei for fibrillation. The $\mathrm{A} \beta$ solution was maintained at $4{ }^{\circ} \mathrm{C}$ to avoid fibril formation. Next, $\mathrm{A} \beta$ fibrils were prepared using the fibril extension method, as previously described [23,24]. Briefly, a stock solution of $\mathrm{A} \beta$ was diluted in Tris buffer $(50 \mathrm{mM})$ at $37^{\circ} \mathrm{C}$ to a final concentration of $10 \mu \mathrm{M}$, which triggered fibril formation. This sample was incubated on a variety of planar membranes 
at $37^{\circ} \mathrm{C}$ for the desired time (up to $120 \mathrm{~h}$ ). As for TIRFM, the fluorescence microscopic system used to observe individual amyloid fibrils was developed based on an inverted microscope (IX70; Olympus, Tokyo, Japan), as described previously [24]. The ThT molecule was excited using an argon laser (model 185F02-ADM; Spectra Physics, Mountain View, CA, USA). The sample solution was mixed with ThT $(5 \mu \mathrm{M})$ and observed using TIRFM.

To estimate fibrillogenesis, fibrils stained by ThT were counted in the field of view in TIRFM. ThT fluorescence intensity was estimated using ImageJ (version 1.32) imaging software. In short, the image was subtracted by the background within the same image, i.e., the place where there was no fibril. Subsequently, the fluorescence intensity originating from ThT bound to amyloid fibrils was extracted and integrated within the image. Afterwards, the integrated fluorescence intensity was averaged using more than ten images.

\subsection{QCM Method}

$A \beta$ accumulation was measured using a QCM [23]. As described in Section 2.2, the planar membrane was immobilized on an AT-cut quartz crystal with a gold electrode (diameter $5.1 \mathrm{~mm}$, resonance frequency $7.995 \mathrm{MHz}$; BAS Inc., Tokyo, Japan). The quartz crystal was connected to ALS/CHI electrochemical QCM equipment (400; BAS Inc.). $A \beta$ solution $(10 \mu \mathrm{M})$ was loaded onto the immobilized membranes to monitor the frequency change. In general, the frequency decrease $(-\Delta f)$ relates to both mass increase per unit area and viscoelastic change. However, as the device used in this study provides only the frequency change, the plateau value of the frequency change was adopted, and not the time course of frequency which sensitively depends on the viscoelastic change.

\subsection{Entanglement Molecular Weight $\left(M_{e}\right)$}

The $M_{\mathrm{e}}$ value was calculated using the following equation [36]:

$$
M_{\mathrm{e}}=\rho R T / G^{0}{ }_{\mathrm{n}},
$$

where $\rho$ is the density of the polymer, $T$ is the temperature, $R$ is the gas constant, and $G^{0}$ is the plateau value at the higher limit of the frequency range of the storage modulus $G_{n}(\omega)$. As the $M_{\mathrm{e}}$ value is generally independent of temperature, the $G^{0}{ }_{n}$ value obtained from rheological measurements using the melting polymer at a given temperature condition could yield the $M_{\mathrm{e}}$ value. The $M_{\mathrm{e}}$ values for the individual polymers are summarized in Table 1.

\section{Results}

\subsection{Fibrillogenesis of $A \beta$ on Each Planar Membrane}

The amyloid fibril formation of $A \beta$ was first examined on each planar membrane. TIRFM permits direct observation of amyloid fibrils stained by ThT, because ThT can specifically bind to fibrils with a rich $\beta$-sheet structure to give its blue-colored fluorescence $[23,24]$. The fibril growth behavior of $A \beta$ on each planar membrane was then examined by TIRFM combined with ThT.

Figure 2A-G shows the TIRFM images of fibrils formed on a variety of planar membranes. As a control (without planar membranes), typical fibrillar aggregates were observed (Figure 2A). In contrast, branched fibrils were observed on the DOPC membrane (Figure 2B). The same was true for the PSf-membranes (Figure 2F). A small number of fibrils was observed on other planar membranes (Figure 2C-E). Interestingly, ring-shaped fibrils were observed on the PLLA-50k-based membranes (Figure 2G, $\mathrm{G}^{\prime}$ ). Such a topology of fibrils might be related to the surface topology of the PLLA-50k-based membranes. The surface was subsequently observed using a scanning electron microscope (SEM). As shown in Figure $2 \mathrm{H}$, a definite pore or pothole structure was formed on the surface. This topology is quite similar to the surface of the PLLA membrane prepared with a casting solution containing 5\% ( $w / w)$ PLLA (85-160 k) [7]. The size of pores present on the surface of the PLLA membranes corresponded to the size of the ring-shaped fibrils. This suggests that the pore structure acted as a template for the formation of ring-shaped fibrils. 

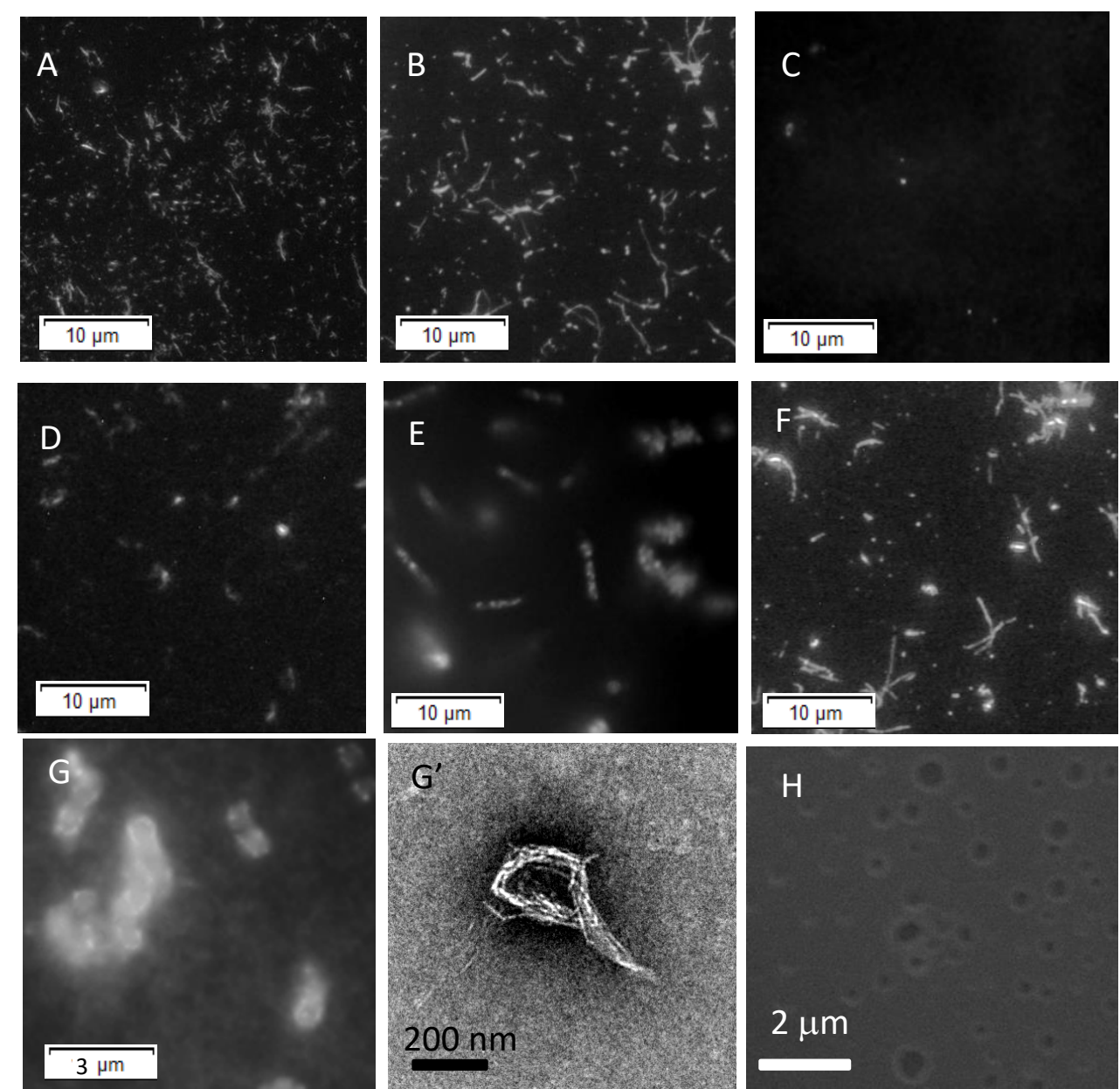

Figure 2. TIRFM images of ThT-stained amyloid fibrils of $A \beta$ on various planar membranes: (A) control; (B) DOPC; (C) iso-PMMA; (D) syn-PMMA; (E) PVP; (F) PSf; (G) PLLA-50k. (G') TEM image of Figure 3G. (H) SEM image of PLLA-50k-based membrane.

\subsection{Kinetic Analysis of A Fibril Growth on Various Membranes}

Variations in the ThT-stained aggregates were monitored for various membranes. To discuss the effect of planar membranes on the kinetic properties of fibril growth, the ThT intensity was plotted against the incubation time for four typical membranes (Figure 3A). The planar membranes showed sigmoidal curves. ThT fluorescence intensity increased at approximately $10 \mathrm{~h}$ in the PSf-based membrane, whereas the PVP-based membrane showed an increase in ThT fluorescence intensity after $80 \mathrm{~h}$. The lag time in the sigmoidal curve corresponds to the nucleation period, and a subsequent increase in ThT intensity represents the elongation of the nuclei [28]. The kinetic parameters associated with the lag time were subsequently assessed using Equation (2):

$$
I(t)=I_{\mathrm{i}}+m_{\mathrm{i}} t+\left(I_{\max }+m_{\mathrm{f}} t\right) /\left(1+\exp \left\{-\left(t-t_{\mathrm{m}}\right) / \tau\right\}\right)
$$

where $t_{\text {lag }}(\mathrm{h})$ is the lag time, $k_{\mathrm{app}}(=1 / \tau)\left(\mathrm{h}^{-1}\right)$ is the apparent elongation rate constant, $I_{\mathrm{i}}$ and $I_{\max }$ are the ThT intensities at the initial and final stages, respectively, and $m_{\mathrm{i}}$ and $m_{\mathrm{f}}$ are the correction factors at the initial and final stages, respectively. The $t_{\mathrm{m}}$ value is associated with the $t_{\text {lag }}$ value as follows: $t_{\text {lag }}=t_{\mathrm{m}}-2 k_{\mathrm{app}}{ }^{-1}$. Thus, the sigmoidal curve shown in Figure $3 \mathrm{~A}$ can be characterized by $k_{\mathrm{app}}, t_{\mathrm{lag}}$, and $I_{\max }$.

The $k_{\text {app }}$ values were plotted against the corresponding $t_{\text {lag }}$ values (Figure $\left.3 \mathrm{~B}\right)$. Overall, a negative correlation was observed between both. This suggests that the fibril formed after the fast nucleation favored fast elongation. In other words, both processes are linked to each other. 
A
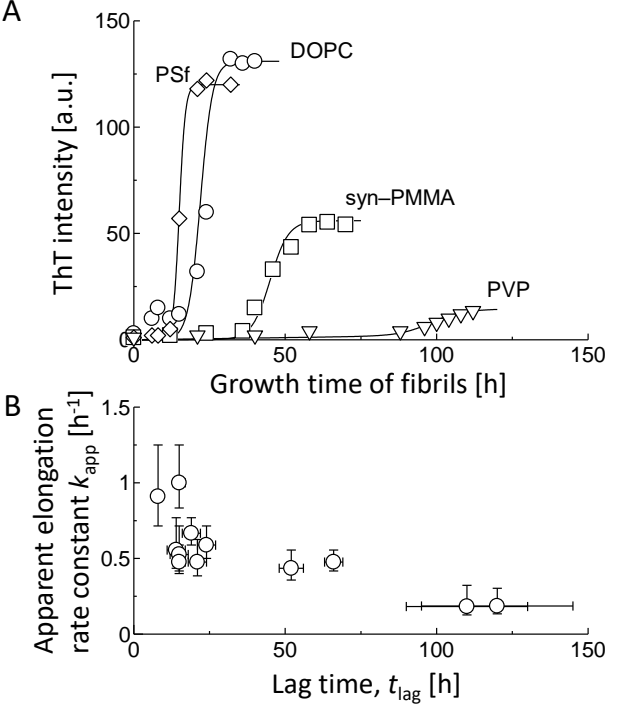
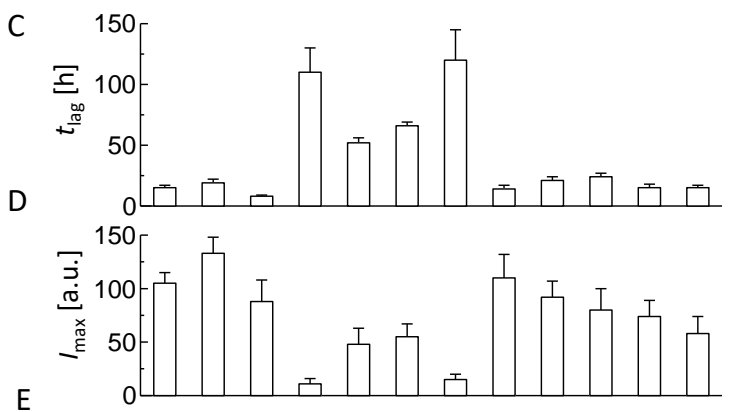

E

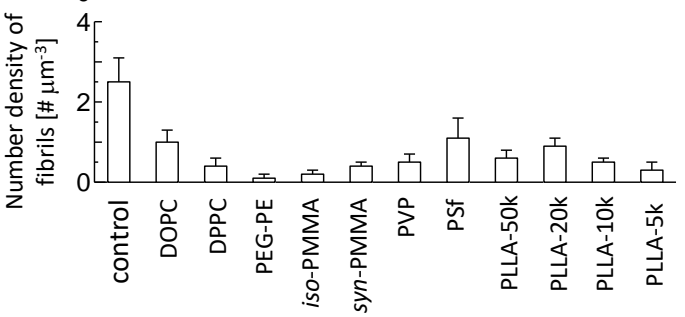

Figure 3. Time-course of (A) ThT fluorescence intensity. Correlation coefficients for four membranes were more than 0.990. (B) Comparison of lag time with apparent elongation rate constant. (C) Lag time, (D) $I_{\max }$, and (E) number density of fibrils on various planar membranes. The concentration of monomeric $\mathrm{A} \beta$ was $10 \mu \mathrm{M}$ in TIRFM observation. Error bars represent the average of experimental data performed at least four times. Error bars represent the average of experimental data performed at least four times.

The $t_{\text {lag }}$ values observed for various planar membranes are shown in Figure 3C. From a comparison of PSf- $(\sim 10 \mathrm{~h})$ with PVP-based membranes $(\sim 120 \mathrm{~h})$, the $t_{\text {lag }}$ value was likely strongly dependent on the hydrophobicity of the polymers. The same was true for PEG-PE-based membranes ( 100 h) considering the induction of hydrophilic PEG chains into phospholipids, such as DOPC and DPPC. The $t_{\text {lag }}$ values for iso-PMMA- and syn-PMMA-based membranes were relatively greater than those for the other membranes, although the influence of tacticity in PMMA was not significant, suggesting that these two membranes were disadvantageous for the nucleation of $A \beta$, as compared with other membranes. In addition, there was no definite influence of the entanglement of polymers on the lag time for PLLAs with different $M w$.

The $I_{\max }$ values, one of the quantitative indices for fibrillogenesis [28], are shown in Figure 3D. $I_{\max }$ for PEG-PE- and PVP-based membranes was very low. PSf-based membranes exhibited high $I_{\max }$ values relative to those of PVP-based membranes. The same was true for DOPC- and DPPC-based membranes relative to the PEG-PE-based membrane. Therefore, the $I_{\max }$ value clearly depends on the hydrophobicity of the planar membranes. The tacticity of PMMA was not significantly observed in $I_{\max }$. Alternatively, the $I_{\max }$ value for PLLA increased with an increase in molecular weight, suggesting a possible influence of the entanglement of polymers. According to a previous report [28], $I_{\max }$ is determined by the number of fibrils formed and/or their nanoscopic structure to which ThT binds. The number density of fibrils after the growth of fibrils was counted subsequently. It may be noted that TIRFM permits the visualization of the evanescent region of $100 \mathrm{~nm}$ in depth. Therefore, the number of fibrils per unit volume of the evanescent region (area of view field $\times$ depth) was defined as the number density of fibrils $\left(\# \mu \mathrm{m}^{-3}\right.$ ). As shown in Figure 3E, the DOPC- and PSf-based membranes had a higher number density of fibrils as compared with PEG-PE- and PVP-based membranes, suggesting the effect of the hydrophobicity of polymers. Other planar membranes had a low number density, giving the impression that there is no definite influence of the tacticity and entanglement of polymers. However, the number density of fibrils indicated a similar trend to the $I_{\max }$ value to some extent. 
From the results, both $t_{\mathrm{lag}}$ and number density of fibrils were selected as kinetic parameters in the following section. This was because both directly reflected the fibrillogenesis of $\mathrm{A} \beta$.

\subsection{A $\beta$ Accumulation on Membranes}

Accumulation of $A \beta$ would be required for the nucleation of $A \beta$ on the planar membrane. The important issue is how the accumulation of $A \beta$ determines fibril growth on planar membranes. Examination of $\mathrm{A} \beta$ accumulation on the planar membranes has been discussed in this section. The QCM method combined with immobilization of lipid membranes is a powerful tool for monitoring the accumulation of $A \beta$ on planar membranes [23]. Accordingly, we evaluated the accumulation of $A \beta$ on the respective planar membranes from the frequency change after the injection of $A \beta(10 \mu \mathrm{M})$.

As shown in Figure 4A, decline in frequency and a subsequent plateau value were observed in the case of phospholipid- and polymer-based membranes. However, in the case of PLLA-5k and PLLA-10k (Figure 4B), the frequency of QCM electrodes decreased initially, followed by an increase up to a positive value relative to the initial state. This was probably because $A \beta$ accumulation on the membranes induced their disruption and detachment from the QCM electrode. In any case, the plateau value of frequency $(\Delta f)$ corresponded to $A \beta$ accumulation on the membranes [23]. The $\Delta f$ values for all the membranes are shown in Figure 4C. For simplification, both PLLA-10k- and PLLA-5k-based membranes, indicating positive values, are depicted as the data (r.c.). For this, the argument concerning the entanglement of polymers was ruled out. The $\Delta f$ values for DOPC-, PSf-, and PLLA50k-based membranes were higher than those for other planar membranes, suggesting the possible contribution of the hydrophobicity of the polymers, rather than other factors, to $\mathrm{A} \beta$ accumulation.

A

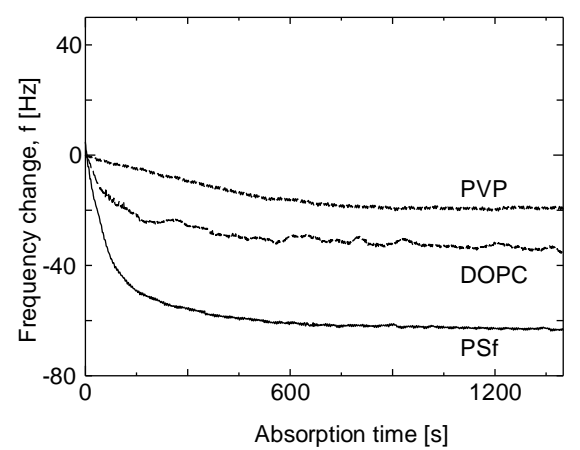

C

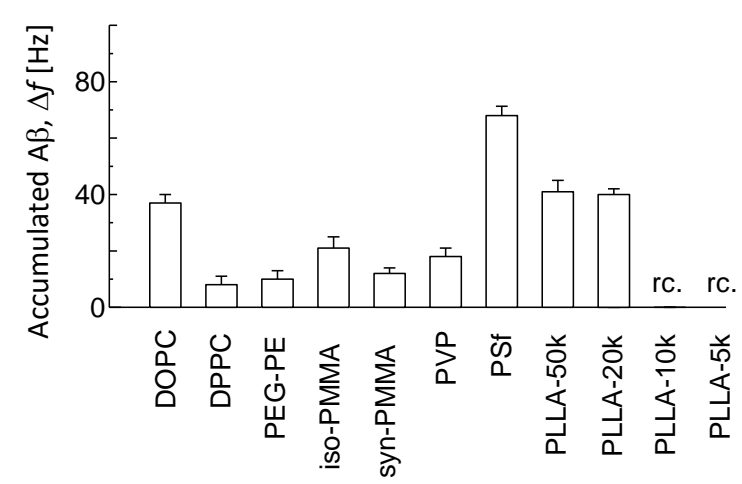

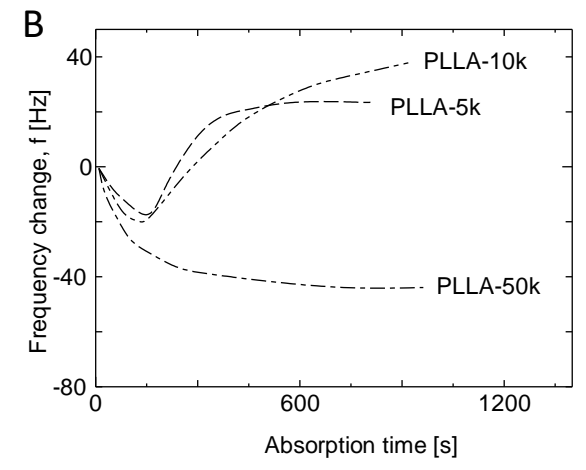

Figure 4. Time course of frequency change of QCM electrodes modified with various kinds of planar membranes after an injection of A $\beta$. (A) DOPC-, PVP-, and PSf-based membranes. (B) PLLA-5k, PLLA-10k, and PLLA-50k-based membranes. Concentration of A $\beta$ injected was $10 \mu \mathrm{M}$. (C) Accumulated amount of $A \beta$ on planar membranes. The "rc." means the recovery behavior of frequency of QCM after the injection of $A \beta$, as indicated in Figure 2B. Error bars represent the standard error of experimental data performed at least four times. 


\subsection{Comparison of $A \beta$ Accumulation with Its Fibril Formation}

$\mathrm{A} \beta$ fibril growth consists of nucleation and subsequent elongation [28]. Further, induction of fibril growth depends on the environment [24,29]. Nucleation of $A \beta$ and its elongation requires its accumulation. The number density of $A \beta$ fibrils formed on each membrane was compared with the corresponding $\Delta f$ value (Figure $5 \mathrm{~A}$ ) to confirm the effect of planar membranes on $A \beta$ fibril growth. Increment in $\Delta f$ increased the number density of the fibrils. It was therefore considered that the accumulation of $A \beta$ resulted in its nucleation. It must be noted that the process involves primary and secondary nucleation [28]. Primary nucleation refers to the nucleation process that occurs in a previously fibril-free solution. In contrast, secondary nucleation is the generation of new fibrils by fibrils already present in the suspension, which is associated with the variation in the number density of fibrils [28]. The present number density of fibrils was attained following both nucleation steps. In this study, the DOPC- and PSf-based membranes indicated a definite variation in the number density of fibrils after the increase in ThT fluorescence intensity (Figure 3A,E). Therefore, primary nucleation is addressed in this study.

A
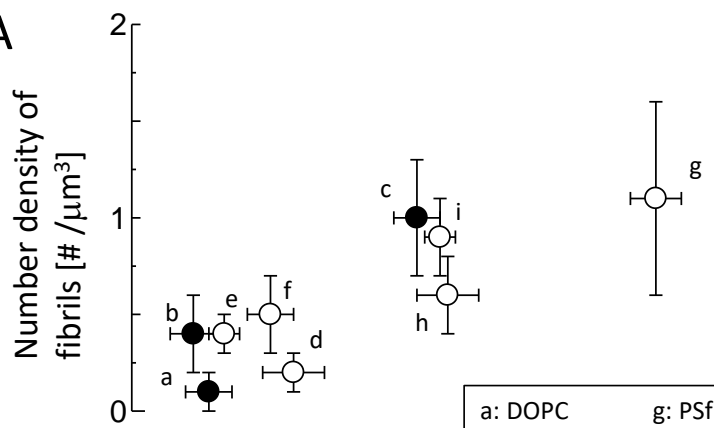

B
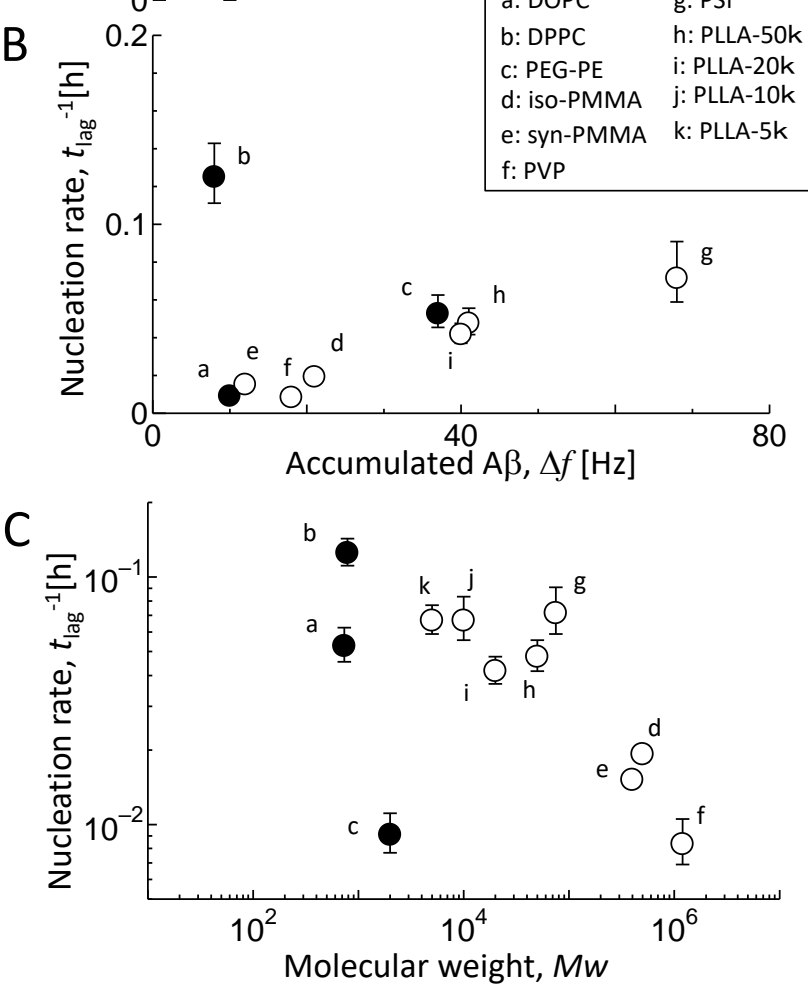

Figure 5. (A) Relationship between the accumulated $\mathrm{A} \beta(\Delta f)$ and its nucleation rate. (B) Relationship between the $\Delta f$ value and the number density of $A \beta$ fibrils. PLLA-5k- and PLLA-10k-based membranes were ruled out in $(\mathbf{A}, \mathbf{B})$. (C) Relationship between the molecular weight of polymers and the nucleation rate. Closed and open keys represent phospholipid- and polymer-based membranes, respectively. 
The rate of primary nucleation can be defined as the reciprocal of the lag time. Accordingly, the nucleation rate of $\mathrm{A} \beta$ was compared with the $\Delta f$ value (Figure $5 \mathrm{~B}$ ). The $\Delta f$ value was linearly proportional to the nucleation rate except for the DPPC-based membrane, i.e., the accumulation of $A \beta$ on planar membranes accelerated its primary nucleation. Thus, the interaction between $A \beta$ and the planar membranes determines the rate of $A \beta$ nucleation.

The interaction between $\mathrm{A} \beta$ and planar membranes is affected by the hydrophobicity of the polymers as well as by the entanglement of polymers [34]. As shown in Table 1, the entanglement depends on the $M w$ of the polymers (see the possible entanglement $M w / M_{\mathrm{e}}$ ). The $M w$ of a polymer also regulates fibril growth [29]. Evans stated that condensed-fluid membranes of lipids behave similarly to linear-flexible polymers [51]. Thus, the rates of $\mathrm{A} \beta$ nucleation were compared with the $M w$ of polymers, by adding the polymers, except PLLA, together with phospholipids (Figure 5C). The increase in $M w$ of the polymer reduced the nucleation rate, except for PEG-PE. It is likely that the deviation of the PEG-PEbased membrane originated from the entanglement by PEG chains. Notably, a correction considering the disruption or detachment of PLLA-5k- and PLLA-10k-based membranes due to their interaction with $\mathrm{A} \beta$ (Figure $4 \mathrm{~B}$ ) would be needed. Thus, an entanglement between polymers and/or between $A \beta$ and the polymer is likely to directly impact the primary nucleation process.

\section{Discussion}

\subsection{Interaction of $A \beta$ with Planar Membranes}

First, we addressed the interaction between monomeric $A \beta$ and planar membranes in terms of electrostatic interactions. Matsuzaki et al. reported that the net charge of monomeric $A \beta$ under physiological conditions was slightly negative [30], which was based on calculations using the $\mathrm{pKa}$ values for $\mathrm{His}^{13}$, $\mathrm{His}^{14}, \mathrm{Glu}^{22}$, and $\mathrm{Asp}^{23}$ of $\mathrm{A} \beta$ with 40 amino acid residues and $\mathrm{Glu}^{3}, \mathrm{Glu}^{11}, \mathrm{His}^{6}, \mathrm{Asp}^{1}, \mathrm{Asp}^{7}$, and $\mathrm{Tyr}^{10}$ of $\mathrm{A} \beta$ with 28 amino acid residues [52,53]. In the present study, the direct electrostatic attractive or repulsive forces between the aforementioned residues and membranes should be small, considering the chemical structure of zwitterionic phospholipids and nonionic polymers. Even in such a state, the electrostatic interaction between the local charge of planar membranes and the individual charges of charged amino acid residues should be considered. For example, PSf-, DOPC-, and PLLA-50k-based membranes showed higher $A \beta$ accumulation than the other membranes (Figure 4C). These membranes were zwitterionic or nonionic, based on their chemical structures (see Figure 1). The choline group in phospholipids has two positive and negative charges. These charges behave individually in the interaction between the phospholipids and $A \beta$. Thus, the interaction of $A \beta$ with these membranes cannot be simply explained by electrostatic interactions.

Alternatively, we discuss the non-electrostatic contribution to the $A \beta$-membrane interaction. $A \beta$ has ten hydrophobic amino acid residues: six Val, two Ile, and two Leu. It is possible that these residues behave individually in hydrophobic interactions. PSf contains a hydrophobic benzene group. Therefore, the hydrophobic interaction between the benzene group in PSf and the hydrophobic transmembrane region of A $\beta$ (Gly29-Val40) with six hydrophobic residues might contribute to the increased accumulation of $A \beta$. In contrast, PVP, PEG-PE, iso-PMME, and syn-PMMA showed low A $\beta$ accumulation. PVP, which is widely used as a powerful hydrophilizing agent, is often blended with a PSf-based membrane owing to its improved hydrophilicity [15]. This hydrophilic property of PVP might be related to its weak $A \beta$ accumulation behavior. In the case of PMMA, a study using sum frequency generation (SFG) vibrational spectroscopy demonstrated the following three aspects [54]: the PMMA surface was dominated by the ester methyl groups; the alpha methyl groups tended to lie down on the PMMA surface; and the methylene groups were not detected on the PMMA surface. In comparison, the surface of the PMMA-based membranes was likely to be hydrophilic. Alternatively, PLLA has a chemical structure similar to PMMA in terms of the inclusion of oxygen in the polymer segment. However, the PLLA-50k-based membrane showed higher A $\beta$ accumulation 
than PMMA-based membranes (Figure 4C). Considering PLLA-5k- and PLLA-10k-based membranes, it is possible that $\mathrm{A} \beta$ formed a complex capable of detachment from the surface of the PLLA-based membranes (Figure 4B). Thus, it is likely that these membranes interact strongly with $\mathrm{A} \beta$. Alternatively, in the case of DOPC and DPPC, both have an identical headgroup, called the phosphocholine group, at the interface of lipid membranes. However, DOPC-based membranes, rather than DPPC-based membranes, showed higher $\mathrm{A} \beta$ accumulation (Figure $4 \mathrm{C}$ ).

From the aforementioned discussion, it may be inferred that only the argument concerning the chemical structure at the interface of polymer and phospholipid membranes provides an insufficient scenario for the interaction of $A \beta$ with the membranes.

\subsection{Contribution of Polymer Entanglement to A $\beta$ Accumulation}

We addressed the topological features of membranes, such as the entanglement of polymers. Polymer-peptide $(\mathrm{A} \beta)$ (i) or polymer-polymer entanglement (ii) may be considered as crucial factors affecting the $A \beta$ accumulation on the membranes. For example, poly(ethylene oxide) ( $M w=5,000,000)$ entangles enough to contribute to protein partitioning [34]. In this section, the effect of entanglement due to the polymer is discussed.

PLLA-5k and PLLA-10k are good examples of case (i). The $M w$ of both polymers is comparable to that of $A \beta$ (approximately 4300). The other polymers used here had a higher $M w$ than A $\beta$. Importantly, only PLLA-5k and PLLA-10k exhibited the recovery of the $\Delta f$ value (Figure $4 \mathrm{~B}$ ). It may be assumed that these membranes were partly disrupted by $\mathrm{A} \beta$, possibly due to polymer-peptide entanglement. Entanglement of $A \beta$ with polymers bearing $M w$ near A $\beta$ might be entropy-driven.

An increase in the length of the polymer backbone significantly increases the possibility of polymer-polymer entanglement (case (ii)). The $M w$ dependency of the nucleation rate (Figure 5C) provides an interpretation with respect to the effect of the entanglement of the polymer. Condensed-fluid membranes of lipids behave similarly to linear-flexible polymers [51]. This is because the lipid molecules move on the solid support solely via lateral diffusion. A study concerning lateral diffusion of DOPC reported that the process was controlled by the entanglement between acyl chains of lipids [55]. Even in the case of DOPC, the nucleation rate was comparable to that of membranes with low Mw. Meanwhile, PEG-PE exhibited a low nucleation rate. Previously, Hashizaki et al. [56] suggested PEG chain-chain entanglement due to van der Waals forces as well as interand intra-chain hydrogen bonds that act in the PEG chains. Thus, PEG chains might interfere with the association of accumulated $A \beta$ on membranes, possibly due to their entanglement. In addition, the surface roughness originating from the PEG chains would act as an obstacle for the association of $A \beta$, because a rough surface made of polystyrene reportedly decelerated the two-dimensional diffusion of $A \beta$ with 42 residues and retarded the fibrillation [33]. In contrast to PEG-PE, polymers with high $M w$, such as iso-PMMA $\left(M w=5.0 \times 10^{5}\right)$, syn-PMMA $\left(M w=4.0 \times 10^{5}\right)$, and PVP $\left(M w=1.2 \times 10^{6}\right)$, also easily entangle in solution, because polymers with large molecular weights generally favor their entanglement, as summarized by $\mathrm{Wu}$ [41]. A possible entanglement of $M w / M_{\mathrm{e}}$ is considerably high, as shown in Table 1 . These polymers reduced the accumulation of $A \beta$ and its nucleation rate in this order.

\subsection{Contribution of the Hydrated Structure of Polymer Membranes}

The influence of entangled polymers becomes prominent in the case of planar membranes. This is because the formation of planar membranes is similar to viscous forced crystallization [43]. Next, we discuss the interaction between $\mathrm{A} \beta$ and planar membranes in terms of the amphiphilicity of $A \beta$. Analysis using an impedance analyzer $[23,44]$ and differential scanning calorimetry [45] suggests that the membrane materials used here favor hydration. For instance, DOPC is hydrated by twenty water molecules per lipid molecule [55]. DPPC also possesses the property of hydration derived from the water of hydration [22]. For PVP, the $-\mathrm{N}-\mathrm{C}=\mathrm{O}$ backbone is hydrated by $5-6 \mathrm{H}_{2} \mathrm{O}$ per unit (see 
Figure 1) [44]. Therefore, it is anticipated that the membrane matrix of PVP is well hydrated. The same must be true for PMMA having a COO group, PSf having $-\mathrm{O}-$ and $-\mathrm{SO}_{2}{ }^{-}$, and PLLA with $-\mathrm{C}=\mathrm{O}$.

Accumulation of $A \beta$ into phospholipid- and polymer-based membranes requires the removal of hydrated water. Nagasawa et al. reported that the thermodynamic cost for the removal of hydrated water in a polymer determines the extent of protein adsorption [16]. This effect likely resulted in the reduced accumulation of $A \beta$ (Figure $4 C$ ) and/or its conformational change, which is advantageous for fibril growth (data not shown). Furthermore, the matrix structure assisted the above effect. The phospholipid- and polymer-based membranes were $5 \mathrm{~nm}$ and at least several tens of nanometers thick, respectively. A $\beta \mathrm{s}$ accumulated on DOPC-based membranes easily bound to each other. In contrast, the matrix of polymer-based membranes likely made the interaction between $A \beta s$ more difficult. Therefore, a well-hydrated PVP-based membrane would afford low A $\beta$ fibrillogenesis.

\section{Conclusions}

We successfully demonstrated that polymer-based membranes can alter the fibrillogenesis of $\mathrm{A} \beta$ and phospholipids. Specifically, PVP, syn-PMMA, and iso-PMMA had a definite impact on fibrillogenesis. This was because both the hydration of polymers and the entanglement between polymers reduced $A \beta$ accumulation on the polymer membranes. PEG-PE also inhibited fibrillogenesis strongly as compared with DOPC and DPPC. This was also due to the hydration and entanglement between the PEG chains. Thus, the hydration and entanglement of polymers had an impact on the fibrillogenesis of $A \beta$. The present findings are considered to have a great potential for applications in the field of biomedicine, such as medical assays and artificial organs, because of the biocompatibility of these polymers.

Author Contributions: Writing—original draft preparation, T.S. and Y.K.; conducting experiments, M.I. and S.D.; analysis, T.S., M.I., and S.D. All authors have read and agreed to the published version of the manuscript.

Funding: This work was supported by Grant-in-Aid KAKEN (26249116) and the Okayama Foundation for Science and Technology (2014).

Institutional Review Board Statement: Not applicable.

Informed Consent Statement: Not applicable.

Acknowledgments: The authors thank TORAY Co. Ltd. for supplying the polymers.

Conflicts of Interest: The authors declare no conflict of interest.

\section{References}

1. Mizuno, H.; Hoshino, J.; So, M.; Kogure, Y.; Fujii, T.; Ubara, Y.; Takaichi, K.; Nakaniwa, T.; Tanaka, H.; Kurisu, G.; et al. Dialysis-related amyloidosis associated with a novel $\beta_{2}$-microglobulin variant. Amyloid 2021, 28, 42-49. [CrossRef]

2. Naiki, H.; Okoshi, T.; Ozawa, D.; Hamaguchi, I.; Hasegawa, K. Molecular pathogenesis of human amyloidosis: Lessons from $\beta_{2}$-microglobulin-related amyloidosis. Pathol. Int. 2016, 66, 193-201. [CrossRef]

3. Kitaguchi, N.; Kawaguchi, K.; Yamazaki, K.; Kawachi, H.; Sakata, M.; Kaneko, M.; Kato, M.; Sakai, K.; Ohashi, N.; Hasegawa, M.; et al. Adsorptive filtration systems for effective removal of blood amyloid $\beta$ : A potential therapy for Alzheimer's disease. J. Artif. Organs 2018, 21, 220-229. [CrossRef] [PubMed]

4. Teodorescu, M.; Bercea, M. Poly(vinylpyrrolidone)—A versatile polymer for biomedical and beyond medical applications. Polym. Plast. Technol. Eng. 2015, 54, 923-943. [CrossRef]

5. Milosavljevic, V.; Jelinkova, P.; Jimenez, A.M.J.; Moulick, A.; Haddad, Y.; Buchtelova, H.; Krizkova, S.; Heger, Z.; Kalina, L.; Richtera, L.; et al. Alternative synthesis route of biocompatible polyvinylpyrrolidone nanoparticles and their effect on pathogenic microorganisms. Mol. Pharm. 2017, 14, 221-233. [CrossRef] [PubMed]

6. Elmowafy, E.M.; Tiboni, M.; Soliman, M.E. Biocompatibility, biodegradation and biomedical applications of poly(lactic acid)/poly(lactic-co-glycolic acid) micro and nanoparticles. J. Pharm. Investig. 2019, 49, 347-380. [CrossRef]

7. Liu, H.-C.; Lee, I.; Wang, J.-H.; Yang, S.-H.; Young, T.-H. Preparation of PLLA membranes with different morphologies for culture of MG-63 Cells. Biomaterials 2004, 25, 4047-4056. [CrossRef] [PubMed] 
8. Punet, X.; Mauchauffe, R.; Rodriguez-Cabello, J.C.; Alonso, M.; Engle, E.; Mateos-Timoneda, M.A. Biomolecular functionalization for enhanced cell-material interactions of poly(methyl methacrylate) surfaces. Regener. Biomater. 2015, 2, 167-175. [CrossRef]

9. Witvrouw, M.; Fikkert, V.; Pluymers, W.; Matthews, B.; Mardel, K.; Schols, D.; Raff, J.; Debyser, Z.; de Clercq, E.; Holan, G.; et al. Polyanionic (i.e., polysulfonate) dendrimers can inhibit the replication of human immunodeficiency virus by interfering with both virus adsorption and later steps (reverse transcriptase/integrase) in the virus replicative cycle. Mol. Pharmacol. 2000, 58, 1100-1108. [CrossRef]

10. Millon, L.E.; Wan, W.K. The polyvinyl alcohol-bacterial cellulose system as a new nanocomposite for biomedical applications. J. Biomed. Mater. Res. Part B 2006, 79B, 245-253. [CrossRef]

11. Ube, T.; Aoki, H.; Ito, S.; Horinaka, J.; Takigawa, T. Relaxation of single polymer chain in binary molecular weight blends observed by scanning near-field optical microscopy. Soft Matter 2012, 8, 5603-5611. [CrossRef]

12. Shen, L.; Adachi, T.; Bout, D.V.; Zhu, X.Y. A mobile precursor determines amyloid- $\beta$ peptide fibril formation at interfaces. J. Am. Chem. Soc. 2012, 134, 14172-14178. [CrossRef] [PubMed]

13. Lim, S.K.; Wong, A.S.W.; de Hong, H.-P.M.; Rangamani, P.; Parikh, A.N.; Nallani, M.; Sandin, S.; Liedberg, B. Spontaneous formation of nanometer scale tubular vesicles in aqueous mixtures of lipid and block copolymer amphiphiles. Soft Matter 2017, 13, 1107-1115. [CrossRef]

14. Radjabian, M.; Abetz, V. Tailored pore sizes in integral asymmetric membranes formed by blends of block copolymers. Adv. Mater. 2015, 27, 352-355. [CrossRef]

15. Hayama, M.; Yamamoto, K.; Kohori, F.; Uesaka, T.; Ueno, Y.; Sugaya, H.; Itagaki, I.; Sakai, K. Nanoscopic behavior of polyvinylpyrrolidone particles on polysulfone/polyvinylpyrrolidone film. Biomaterials 2004, 25, 1019-1028. [CrossRef]

16. Nagasawa, D.; Azuma, T.; Noguchi, H.; Uosaki, K.; Takai, M. Role of interfacial water in protein adsorption onto polymer brushes as studied by SFG spectroscopy and QCM. J. Phys. Chem. C 2015, 119, 17193-17201. [CrossRef]

17. Mattson, M.P. Pathways towards and away from Alzheimer's disease. Nature 2004, 430, 631-639. [CrossRef]

18. Hardy, J.; Selkoe, D.J. The amyloid hypothesis of Alzheimer's disease: Progress and problem on the road to therapeutics. Science 2002, 297, 353-356. [CrossRef]

19. Lee, V.M.-Y.; Trojanowski, J.Q. Mechanisms of Parkinson's disease linked to pathological $\alpha$-synuclein: New targets for drug discovery. Neuron 2006, 52, 33-38. [CrossRef]

20. Guo, T.; Zhang, D.; Zeng, Y.; Huang, T.Y.; Xu, H.; Zhao, Y. Molecular and cellular mechanisms underlying the pathogenesis of Alzheimer's disease. Mol. Neurodegener. 2020, 15, 40. [CrossRef] [PubMed]

21. Hane, F.; Leonenko, Z. Effect of metals on kinetic pathways of amyloid- $\beta$ aggregation. Biomolecules 2014, 4, 101-116. [CrossRef]

22. Sabate, R.; Estelrich, J. Evidence of the existence of micelles in the fibrillogenesis of beta-amyloid peptide. J. Phys. Chem. B 2005, 109, 11027-11032. [CrossRef]

23. Shimanouchi, T.; Nishiyama, K.; Hiroiwa, A.; Vu, H.T.; Kitaura, N.; Umakoshi, H.; Kuboi, R. Growth behavior of A $\beta$ protofibrils on liposome membranes and their membrane perturbation effect. Biochem. Eng. J. 2013, 71, 81-88. [CrossRef]

24. Yagi, H.; Ban, T.; Morigaki, K.; Naiki, H.; Goto, Y. Visualization and classification of amyloid $\beta$ supramolecular assemblies. Biochemistry 2007, 46, 15009-15017. [CrossRef]

25. Krebs, M.R.H.; MacPhee, C.E.; Miller, A.F.; Dunlop, I.E.; Dobson, C.M.; Donald, A.M. The formation of spherulites by amyloid fibrils of bovine insulin. Proc. Natl. Acad. Sci. USA 2004, 101, 14420-14424. [CrossRef] [PubMed]

26. Krebs, M.R.H.; Bromley, E.H.C.; Rogers, S.S.; Donald, A.M. The mechanism of amyloid spherulite formation by bovine insulin. Biophys. J. 2005, 88, 2013-2021. [CrossRef] [PubMed]

27. Shimanouchi, T.; Shimauchi, N.; Onishi, R.; Kitaura, N.; Yagi, H.; Goto, Y.; Umakoshi, H.; Kuboi, R. Formation of spherulitic amyloid $\beta$ aggregate by anionic liposomes. Biochem. Biophys. Res. Comm. 2012, 426, 165-171. [CrossRef]

28. Shimanouchi, T.; Kitaura, N.; Onishi, R.; Umakoshi, H.; Kuboi, R. Secondary nucleation of A $\beta$ fibrils on liposome membrane. AIChE J. 2012, 58, 3625-3632. [CrossRef]

29. Ban, T.; Morigaki, M.K.; Yagi, H.; Kawasaki, T.; Kobayashi, A.; Yuba, S.; Naiki, H.; Goto, Y. Real-time and single fibril observation of the formation of amyloid $\beta$ spherulitic structure. J. Biol. Chem. 2006, 281, 33677-33683. [CrossRef]

30. Ikeda, K.; Matsuzaki, K. Driving force of binding of amyloid $\beta$-protein to lipid bilayers. Biochem. Biophys. Res. Comm. 2008, 370, 525-529. [CrossRef]

31. Matsuzaki, K. Physicochemical interactions of amyloid $\beta$-peptide with lipid bilayers. Biochim. Biophys. Acta 2007, 1768, 1935-1942. [CrossRef] [PubMed]

32. Kuboi, R.; Shimanouchi, T.; Yoshimoto, M.; Umakoshi, H. Detection of conformational change of protein under stress conditions. Sens. Mater. 2004, 16, 241-254.

33. Shezad, K.; Zhang, K.; Hussain, M.; Dong, H.; He, C.; Gong, X.; Xie, X.; Zhu, J.; Shen, L. Surface roughness modulates diffusion and fibrillation of amyloid- $\beta$ peptide. Langmuir 2016, 32, 8238-8244. [CrossRef] [PubMed]

34. Abbott, N.L.; Blankschtein, D.; Hatton, T.A. Protein partitioning in two-phase aqueous polymer systems. 4. Protein in solutions of entangled polymers. Macromolecules 1992, 25, 5192-5200. [CrossRef]

35. Nishio, Y.; Haratani, T.; Takahashi, T. Miscibility and orientation behavior of poly(vinyl alcohol)/poly(vinyl pyrrolidone) blends. J. Polym. Sci. Polym. Phys. 1990, 28, 355-376. [CrossRef]

36. Ferry, J.D. Viscoelastic Properties of Polymers; Wiley: New York, NY, USA, 1980. 
37. Litvinov, V.M.; Ries, M.E.; Baughman, T.W.; Henke, A.; Matloka, P.P. Chain entanglements in polyethylene melts. Why is it studied again? Macromolecules 2013, 46, 541-547. [CrossRef]

38. Kajiyama, T.; Tanaka, K.; Takahara, A. Surface thermal molecular motion of chain end-modified polystyrenes. Macromol. Symp. 2003, 192, 265-270. [CrossRef]

39. Dalvi, M.C.; Eastman, C.E.; Lodge, T.P. Diffusion in microstructured block copolymers: Chain localization and entanglements Phys. Rev. Lett. 1993, 71, 2591-2594. [CrossRef] [PubMed]

40. Wang, S.Q. Chain dynamics in entangled polymers: Diffusion versus rheology and their comparison. J. Polym. Sci. Part B Polm. Phys. 2003, 41, 1589-1604. [CrossRef]

41. Chun, J.-Y.; Choi, M.-J.; Min, S.-G.; Weiss, J. Formation and stability of multiple-layered liposomes by layer-by-layer electrostatic deposition of biopolymers. Food Hydrocoll. 2013, 30, 249-257. [CrossRef]

42. Wu, S. The origin of chain entanglement: Correlations between entanglement and chain structure. Polym. Eng. Sci. 1993, 33, 289-292. [CrossRef]

43. Yamazaki, S.; Gu, F.; Watanabe, K.; Okada, K.; Toda, A.; Hikosaka, M. Two-step formation of entanglement from disentangled polymer melt detected by using nucleation rate. Polymer 2006, 47, 6422-6428. [CrossRef]

44. Shinyashiki, N.; Matsumura, Y.; Miura, N.; Yagihara, S.; Mashimo, S. Dielectric study of water structure in polymer solution. J. Phys. Chem. 1994, 98, 13612-13615. [CrossRef]

45. Bach, D.; Miller, I.R. Hydration of phospholipid bilayers in the presence and absence of cholesterol. Chem. Phys. Lipids 2005, 136, 67-72. [CrossRef]

46. Yamamoto, T.; Furukawa, H. Prediction of mechanical properties of glassy polymers by group contribution method. Kobunnshi Ronbunnsyu 1995, 52, 187-193. [CrossRef]

47. Wu, S. Chain structure and entanglement. J. Polym. Sci. B Polym. Phys. 1989, 27, 723-741. [CrossRef]

48. Dorgan, J.R.; Williams, J.S. Melt rheology of poly(lactic acid): Entanglement and chain architecture effects. J. Rheol. 1999, 43, 1141-1155. [CrossRef]

49. Cremer, P.S.; Boxer, S.G. Formation and spreading of lipid bilayers on planar glass supports. J. Phys. Chem. B 1999, 103, 2554-2559. [CrossRef]

50. Mouhamad, Y.; Mokarian-Tabari, P.; Clarke, N.; Jones, R.A.L.; Geoghegan, M. Dynamics of polymer film formation during spin coating. J. Appl. Phys. 2014, 116, 123513. [CrossRef]

51. Evans, E. Entropy-driven tension in vesicle membranes and unbinding of adherent vesicles. Langmuir 1991, 7, 1900-1908. [CrossRef]

52. Zhang, S.; Lee, J.P. Selectively ${ }^{2}$ H-labeled Glu/Asp: Application to $\mathrm{pK}_{\mathrm{a}}$ measurement in $\mathrm{A} \beta$ amyloid peptide. J. Pept. Res. 2000, 55, 1-6. [CrossRef]

53. Ma, K.; Clancy, E.L.; Zhang, Y.; Ray, D.G.; Wollenberg, K.; Zagorski, M.G.J. Residue-specific pK $\mathrm{a}_{\mathrm{a}}$ measurements of the $\beta$-peptide and mechanism of $\mathrm{pH}$-induced amyloid formation. J. Am. Chem. Soc. 1999, 121, 8698-8706. [CrossRef]

54. Wang, J.; Chen, C.; Buck, S.M.; Chen, Z. Molecular chemical structure on poly(methyl methacrylate) (PMMA) surface studies by sum frequency generation (SFG) vibrational spectroscopy. J. Phys. Chem. B 2001, 105, 12118-12125.(54). [CrossRef]

55. Filippov, A.; Oradd, G.; Lindblom, G. Influence of cholesterol and water content on phospholipid lateral diffusion in bilayers. Langmuir 2003, 19, 6397-6400. [CrossRef]

56. Hashizaki, K.; Taguchi, H.; Itoh, C.; Sakai, H.; Abe, M.; Saito, Y.; Ogawa, N. Effects of poly(ethylene glycol) (PEG) chain length of PEG-lipid on the permeability of liposomal bilayer membranes. Chem. Pharm. Bull. 2003, 51, 815-820. [CrossRef] 